\title{
Study on Reasonable Injection-Production Parameters of Water- Flooding Reservoir: A Case Study of Daqing Oilfield
}

\author{
Cong Nie \\ The Sixth Oil Production Plant, Daqing Oilfield Co., Ltd., Daqing 163114, Heilongjiang, China
}

\begin{abstract}
This paper, by conducting comprehensive analysis on water-flooding reservoir, intensively studied and discussed the characteristics of geological development, determined the evaluation indexes of development effect, carried out research from five steps, the first is the introduction part, it conducted indepth study and discussion from research background, research significance, literature review, and research methods four aspects. The second outlined the related theory from the optimization principle and mechanism of injection-production parameters, and the distribution mechanism of remaining oil two aspects. The third analyzed the current situation of reasonable injection-production of water-flooding reservoirs in Daqing Oilfield from reservoir characteristics and reservoir deposition. The fourth evaluated the water-flooding development effect from combined water cut, changing conditions of production and predicted recovery ratio. The fifth intensively studied the reasonable injection-production parameters of water- flooding reservoirs in Daqing oilfield from design of separated layer water injection plan, maintaining level reservoir formation pressure, and reasonable plan of injection-production ratio and oil recovery rate three aspects, and provides theoretical guidance for later targeted excavation of water-flooding reservoirs.
\end{abstract}

Keywords: Water-flooding reservoir; injection-production parameters; Daqing oil field; structural characteristics.

\section{Analysis of the Current Situation of Reasonable Injection-Production of Water-Flooding Reservoirs in Daqing Oilfield}

\subsection{Survey of Daqing Oilfield}

Daqing Oilfield was discovered in 1959 and started to develop in 1960, Daqing Oilfield is the largest oilfield in China, and it is one of the largest continental sandstone oilfields in the world. Daqing Oilfield is located in the northern part of the Songnen Plain and the middle and western part of Heilongjiang, the oil and gas in this oil field consists of oil and gas in Lamadian, Hailar, Chaoyanggou, Saertu, Xingshugang and so on. There are more than 90000 oil and gas wells in Daqing Oilfield. Generally speaking, after fracture, the faults in Daqing Oilfield mainly contain oil and gas layers, they extend upward and cross the cap rock and oil layer of group I and group II of Nenjiang River, and some even extend to the area of Mingshui group. Due to the channel nature of the faulted water layer, oil and gas can move upward, they can be stored in the top of the section II of Nengjiang, the section III of Nengjiang and the section V of Nengjiang area, and even reach the Mingshui group, thus forming the secondary oil and gas reservoir. They are still controlled by the structure. The reservoir types in the Daqing Oilfield are relatively simple, due to the influence of anticlinal structural factors, it plays an important role in the formation of oil and gas reservoirs in this area, due to the influence of faults, lithology and other factors, it also has a certain impact in some positions, then structural oil-gas reservoirs and different types of composite oil-gas reservoirs are formed.

\subsection{Characteristics of reservoir deposit}

The production oil layers of Daqing oil field have the following layers: the first is the Putaohua oil layer. This layer belongs to the important containing layer in the southern of Daqing oil field; in addition, there are mainly fine sands in the southern of Aobaota tip. The average porosity is $17 \%$ to $20 \%$, the thickness of fine sands is generally 9 to $18 \mathrm{~m}$, the permeability is $10-3 \mathrm{~m}^{2} \times(100-240)$. The second is the Salto oil layer. This layer is the main production layer of the oil field, this layer is mostly composed of medium sandstone and fine sandstone, this layer mainly includes four storage oil groups, they are mainly S0, S1, S2, and S3. The northern S2 group of the oil field belongs to the medium prototype river deposition; the shape of the single sand body is geometrical, presents 
the belt distribution to the south of Changyuan, presents the shape of sandstone lens body, and it is deposited in the front facies of the delta. The type of sand layer in the area, its thickness is $5-10 \mathrm{~m}$, the average porosity of this layer is $18 \%-20 \%$, and its permeability is $10-\mathrm{m}^{2} \times 100$, this area belongs to permeable oil reservoir. The third is Gaotaizi oil layer. This layer is mainly distributed in the northern area of Daqing oil field, its porosity is $18 \%$ to $26 \%$, the thickness of its single layer is only about $1 \mathrm{~m}$, and the permeability is $10-3 \mathrm{~m}^{2} \times(50 \sim 350)$.

\section{Effect Evaluation of Water-Flooding Reservoir}

This paper studies the side energy water of Daqing Oilfield, the amount of water is weak, natural energy is weak, stratum pressure and saturation pressure is relatively small, self-spurt capacity of oil wells, if we want to obtain better collection effect, we should supplement the energy of stratum by artificial way. Since it is more convenient to obtain water resources on water, and it has the advantages of water-flooding development technology and economic maturity, therefore, water injection development must be used to collect. However, the water-flooding process is complicated due to the influence of geological factors and development factors. The following conducts comprehensive evaluation for the existing geological development data and development effect of oil field, its evaluation is also to provide a certain theoretical basis for the appropriate adjustment of oil field development later. There are many approaches to evaluate the oilfield development, which mainly include the multiindex evaluation comprehensive evaluation approach and the single-index evaluation approach, and they contain the integrated water content, production change situation, predicted recovery rate and other aspects of contents.

\subsection{Comprehensive Water Content}

Comprehensive water content evaluation is a comprehensive concept, which can reflect the water content of crude oil in oilfield and the development effect of the oilfield. When a field which is developed with water-flooding and reaches a high water content stage, the comprehensive water content evaluation approach plays an important role in adjusting the field development plan and studying the field development dynamics.

\subsubsection{Longitudinal Distribution of Water Content}

Due to the shallow burial depth, many stratums, and thick oil layer in test area, and the longitudinal inhomogeneity of the reservoir is more serious. In addition, there is a certain difference in the watered-out degree in the longitudinal direction of the reservoir. Therefore, there are large differences in the development effect of water flooding. The author used eclipse numerical simulation software for the longitudinal distribution evaluation of water content in the study area, divide the reservoir water flooding longitudinally in accordance with sections, the vertical distribution of water flooding in each main oil layer can be obtained, as shown in Fig.1. It can be concluded that the water content in the oil layer group is generally high via specific analysis and research. The water content of each oil layer group is relatively low, and the layered water injection amount is adjusted appropriately based on the water content of different levels, so achieve the purpose of improving the development water-flooding effect.

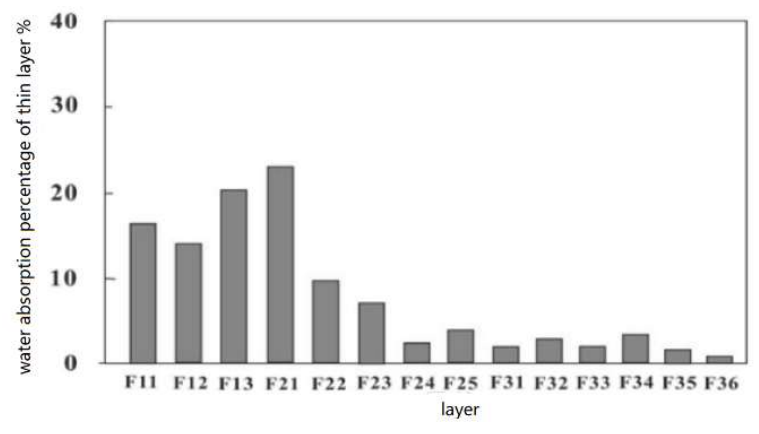

Fig.1 Water content distribution in the test area

\subsubsection{Relationship between Recovery Layer and Water Content}

At present, the commonly used methods to analyze the relationship between the recovery layer and water content have flow pipe method, phase permeation curve method and Tong's calibration curve method. Relatively speaking, the principle of phase permeation curve method is relatively simple and quick to calculate, and it is a common method in reservoir engineering. According to the curve, it can be seen visually that different adjustment methods can be used for different conditions at different time points, and the different adjustment effect can be obtained. The development effect at different stages can be correctly evaluated by effectively comparing the theoretical curve with the actual curve. In addition, it is also possible to predict the condition of recovery ratio. The prediction for the test area can obtain the water content, by comparing the change curve between standard chart and recovery degree, as shown in Fig.2. There are mainly five curves within the standard chart, the reservoir replacement characteristics and oil storage properties of each curve are somewhat different, the relationship among the gap structure, wettability, and fluid properties of reservoir rock are comprehensively analyzed. The formula relationship:

Formula 1: convex curve: $\mathrm{R}=\mathrm{B}\left(\ln -f_{-} \mathrm{w}\right)$

Formula 2: the transition curve between S-type and curved: $A+B\left(\ln -f_{-} w\right)=\ln (1-R)$

Formula 3: the transition curve between concave line $\mathrm{S}$ type: $\mathrm{A}+\mathrm{B}\left(\ln 1-f_{-} \mathrm{w}\right)=\ln \mathrm{R}$

Formula 4: S-type curve is $\mathrm{R}: \mathrm{R}=\mathrm{B}\left\{\ln 1-f_{-} \mathrm{w} /\left(1-f_{-} \mathrm{w}\right)\right\}+\mathrm{A}$ Formula 5: concave curve: $\ln \mathrm{R}=\mathrm{B} \ln f_{-} \mathrm{w}+\mathrm{A}$ 


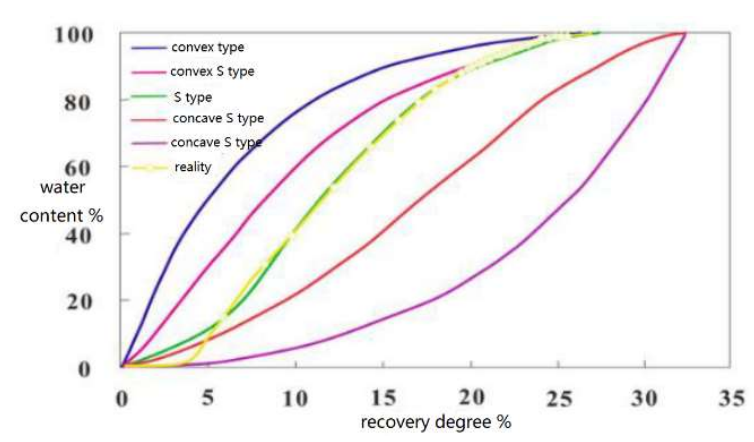

Fig.2 Relationship curve between the recovery degree and water content

At present, by drawing recovery degree and standard charts of water content, and comparing them with relationship curves, we can conclude that the curve is consistent with the concave shape in the early stage, and roughly keep with the $\mathrm{S}$ type in the later stage, the water content rises quickly in the early stage. This is because the reservoir type in the test area is a highly porous and highly permeable oil layer. When water is injected into the oil wells, the concentration of oil and water is relatively large and the water content rises fast, so we can conclude that most of the reservoir reserves are recovered from the high water content period.

\subsection{Changing Conditions of Output}

The evaluation of the dimensionless fluid recovery and oil recovery index can be used to obtain the changing conditions of oil amount. First, the actual fluid parameters, reservoir physical properties and phase permeability curves of the test area are applied to the mathematical model of oil recovery index, and the relationship among dimensionless fluid recovery, oil recovery index and water content in the test area is drawn, as shown in Fig.3. The dimensionless fluid recovery index in the test area continuously increases as water content rises; in addition, the dimensionless recovery index continuously increases as water content rise, its variation is relatively large. This is because the study area actually belongs to the reservoir with high oil-water viscosity and high permeability. When the flow pressure and stratum pressure are stable, the index of dimensionless fluid recovery will be influenced by the oil-water viscosity and permeability, it will and more severe. In general, the permeability will affect the changing trend of the dimensionless fluid recovery index, but the oil-water viscosity will affect the dimensionless fluid recovery index, so there will be a large upward trend in the high water-bearing period.

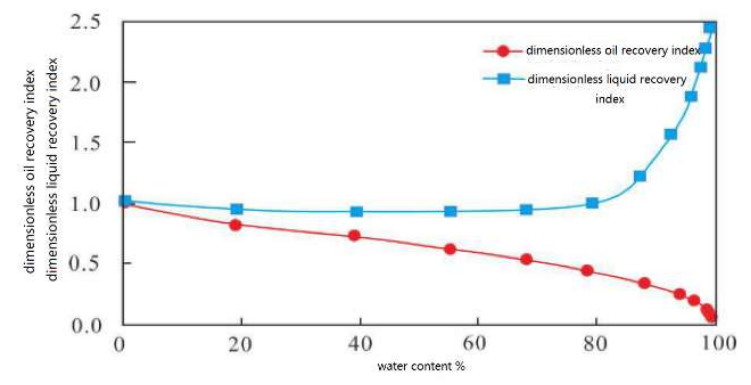

Fig.3 Relationship curve among water content, dimensionless oil recovery and liquid recovery

\subsection{Predicted Recovery Ratio}

The development level of the oil field can be measured by collecting the pre-recovery ratio, which is influenced by the reservoir management level, structural characteristics, extraction mode, extraction technology and other relevant factors. According to the reservoir characteristics of the oil field, more prediction methods of recovery ratio will be generated. At present, the more commonly used methods are water-flooding law curve method, empirical formula method, water-flooding oil experiment method, core analysis method, etc. In the following evaluation of oil collection condition, the water-flooding law curve method is mainly used, and theoretical derivation is used to predict the recovery ratio of the test area.

The water-flooding law curve method is mainly based on the oil-water two-phase permeation mechanism, creates the mathematical relationship based on the production law, and the quantitative relationship of production data is built by using regression curves in the median coordinate system, the recovery ratio conditions during the limit water cut period can be obtained. The test area of this study belongs to layered reservoir with high crude oil viscosity. According to the curve selection criteria of water flooding, the regular tendency of water flooding of type B can be collected. Predecessors obtained the half pair curve relationship between cumulative oil production and cumulative liquid production via the specific form of empirical formula, later, it was uniformly named type B water flooding. The following is the specific expression formula.

$$
\log L_{P}=\alpha+\mathrm{bN}_{P}
$$

In the formula. $\alpha, b$ are regression constants and are dimensionless. $L_{P}$ is the cumulative fluid production $10^{4} \mathrm{t}$. The relation between water content and cumulative oil production: $N_{P}=\frac{\log \left(\frac{1}{1 f_{w}}\right)-c}{b}$

In the formula: $c=\alpha+\log \quad(2.303 . b)$

If in the state of extreme water content, therefore, the formula of the amount of reserves that can be recovered can be obtained:

$$
\mathrm{N} N_{\text {lim }}=\frac{\log \left(\frac{1}{1 f_{\text {wlim }}}\right)-[\alpha+\log (2.303 \mathrm{~b})]}{b}
$$

Taking the specific experimental area as an example, the water drive law curve of type B is effectively fitted, and the fitted law which we come to is shown in Fig.4. 


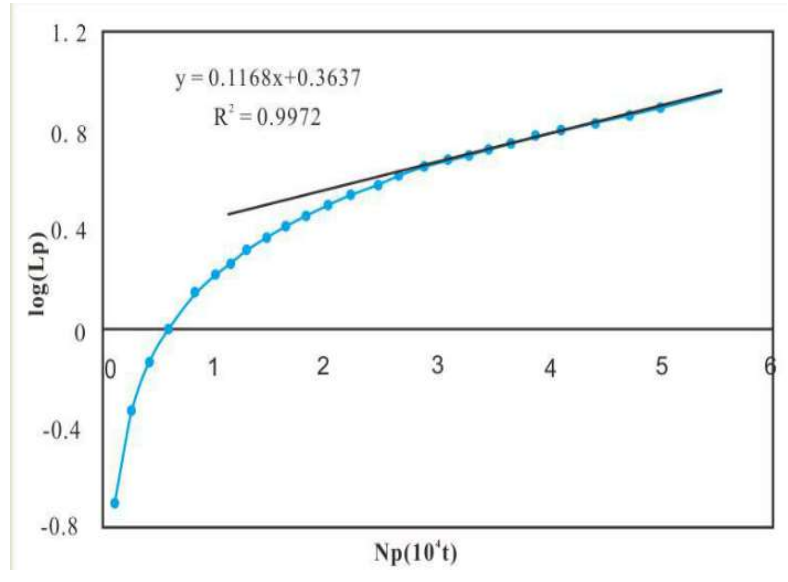

Fig.4 Curve of B-type water-flooding law in the test area

Table 1. Gets the calculation results of predicted recovery ratio of water-flooding law curve in the test area.

\begin{tabular}{|c|c|c|c|c|}
\hline \multirow{2}{*}{ parameter } & $\mathrm{a}$ & $\mathrm{b}$ & $\begin{array}{c}\text { recovery degree } \% \\
\text { content } \\
95 \%\end{array}$ & $\begin{array}{c}\text { water } \\
\text { content } \\
90 \%\end{array}$ \\
\hline $\begin{array}{c}\text { numerical } \\
\text { value }\end{array}$ & 0.3635 & 0.1166 & 28.84 & 26.99 \\
\hline
\end{tabular}

According to the above table, when the extreme water content reaches $90 \%$, the predicted recovery ratio is $28.84 \%$, and when the extreme water content reaches $95 \%$, its predicted recovery ratio is $28.84 \%$.

\section{Conclusion}

In conclusion, the development of oilfield projects is the key contents of national project, and its research and construction is an important content in promoting the sustainable development of China's petroleum cause. Moreover, the development of water-flooding reservoirs in our country has entered into the middle and late stage, many problems and contradictions have gradually emerged, this causes the relevant departments to deepen their exploration of the development law of waterflooding reservoirs, and continuously study waterflooding reservoirs as well as water-flooding reservoirs with good water content in depth, and then help people better solve the problem of the conflict between supply and demand.

\section{References}

1. Lv Duanchuan, Wu Wei. Injection-Production Parameter Adjustment of Water Flooding Development: Taking Two Fault-Blocks on Both Sides of F Fault of Daqing Oilfield Bei'Erxi as Examples[J]. Fault-Block Oil \& Gas Field, 2017(06):83-86.

2. Huang Bin, Xu Rui, Song Kaoping, Fu Cheng, Zhang Wei, Shi Zhenzhong. Identification of Thief Iones Between Injection Wells and Production Wells of
Standstone Reservoirs Flooding by Fuzzy Comprehensive Analysis Method[J]. Mathematics in Practice and Theory, 2017(13), 127-135.

3. Long Ming, $\mathrm{Xu}$ Yanan, $\mathrm{Yu}$ Dengfei, et al. Quantitative Optimization of Water Injection for Bohai Heavy Oil Reservoir with Bottom Water: A Case Study of the West Block of QHD32-6 Oilfield [J]. China Offshore Oil and Gas, 2017(4), 91-97.

4. Li Shujing, Zhang Tianjie, Yang Jian, et al. Study on Prediction Method of Reasonable Injection-toProduction Ratio in High Water-cut Stage of Yan'an Formation Reservoir: Taking A Reservoir in Ansai Oilfield as an Example[J]. Technology Supervision in Petroleum Industry, 2017 (10), 12-14.

5. Wang Langbo, Gao Xiangrui, Dai Bo, et al. Study on Single Sand Body in Ultra-Low Permeability Reservoir and Adjustment to Injection-Production: An Example from Wangyao Area of Ansai Oilfield[J]. Journal of Petrochemical University, 2018, 31 (2): 82-88.

6. Zhang Jingtao. Optimization of Injection-Production Parameters and Development Index of Mixed Well Pattern in Daqing M15-1 Block[D]. Yanshan University, 2016.

7. Shi Pengtao, Chen Penggang. Study on Reasonable Injection-Production Ratio of Wangjiawan Chang 2 Reservoir [J]. Petrochemical Industry Application, 2017(04): 82-83,93. 